\title{
Attività normativa: pubblicate le norme UNI per geotermia, in corso il progetto di norma per i pozzi.
}

\author{
Stefano Chiarugi \\ Associazione Acque Sotterranee - ANIPA - Piacenza \\ stefanochiarugi@landipozzi.it
}

Un piccolo gruppo di associati, sollecitato dal Consiglio dell'ANIPA, ha dato il proprio contributo alla stesura del pacchetto di norme UNI per gli "impianti a pompa di calore" e per i "pozzi per acqua”. Ancor più, sul tema dei pozzi per acqua, la nostra Associazione ha promosso in prima persona l'attività normativa.

Il lavoro si è svolto sotto la regia del Comitato Termotecnico Italiano, vera fucina di produzione dei progetti normativi nell'ambito dell'energia termica in generale e che nel nostro caso specifico è stato "prestato alla risorsa idrica sotterranea" in virtù della grande capacità tecnica ed esperienza nel processo che porta alla pubblicazione della Norma UNI.

\section{Geotermia a pompa di calore}

L'attività è in stato avanzato di sviluppo con tre Norme già pubblicate, un progetto concluso e uno i fase di elaborazione, altri in programma. Come sappiamo le norme UNI sono disponibili solo con l'acquisto ed è vietata la riproduzione. Ci limitiamo quindi al commento di alcuni contenuti rimandando i particolari alla lettura delle norme stesse.

Le tre norme pubblicate riguardano:

- $\quad$ Sistemi geotermici a pompa di calore - Requisiti per il dimensionamento e la progettazione UNI 11466:2012

- Sistemi geotermici a pompa di calore - Requisiti per l'installazione UNI 11467:2012

- Sistemi geotermici a pompa di calore - Requisiti ambientali UNI 11468:2012

Esaminando solo gli aspetti di nostra competenza, possiamo osservare che la norma sull'installazione contiene alcuni elementi di novità procedurale. Essa descrive, fra l'altro, le attività relative alla costruzione di sonde geotermiche e dei pozzi di presa e di resa: è evidente che trattandosi di una norma non tratta di novità tecnologiche, ma si limita a fotografare lo stato dell'arte, ciò nonostante introduce importanti novità di approccio ai problemi che gli operatori si trovano ad affrontare.

\section{Sonde geotermiche}

Nella parte che tratta delle tecniche di perforazione si espongono tutte le tecnologie possibili (doppia testa, circolazione diretta, martello fondo foro) indicando gli ambiti di applicazione ottimale. In questo modo si sgombra il campo da argomenti senza fondamento tecnico quale l'assunta prerogativa di non contaminare gli acquiferi da parte della tecnologia con doppia testa. In effetti eseguire perforazione con tubi di rivestimento minimizza i fenomeni di fuga dei fluidi nelle formazioni attraversate, ma non garantisce "di per se" l'isolamento degli acquiferi nella fase di recupero del rivestimento e contemporanea esecuzione della cementazione. A questo proposito la norma definisce con precisione le "modalità di riempimento delle sonde verticali" (cioè la cementazione) $\mathrm{e}$ in un allegato si propone un modello di verbale che impresa e DL debbono sottoscrivere. La procedura descritta, l'unica in grado di certificare l'efficacia delle operazioni effettuate, è delicata e onerosa, ha però il pregio di definire le responsabilità degli operatori. Ritengo che ciò non risolva il problema, infatti questa procedura, guarda caso nelle situazioni critiche di acquiferi sovrapposti ad elevata permeabilità, o è rispettata in modo pedissequo e quindi diviene molto onerosa oppure non garantisce circa la possibilità di eseguire opere che non provochino danno agli acquiferi. E' auspicabile un intervento legislativo che, come negli altri paesi europei, eviti la realizzazione di opere in contesti "sensibili", dato che le regole del mercato non sono, da sole, capaci di governare questa criticità.

\section{Pozzi di presa e resa}

Anche sull'argomento degli impianti geotermici a circuito aperto ci sono importanti elementi che consolidano prassi correnti positive.

La scelta delle tecniche di perforazione è correlata alla tipologia di terreno da perforare e non vengono poste condizioni preconcette. Chiarito che non esistono problematiche di danneggiamento irrisolvibile a fronte di una buona conduzione delle varie tecniche di perforazione, vengono evidenziati obbiettivi e procedure per garantire il committente da responsabilità nei confronti di terzi e dell'ambiente. Il ruolo del progettista è decisivo nel valutare nel merito l'applicabilità e l'efficacia delle tecniche adottate.

La facoltà di valutare il risultato dell'opera, cioè l'efficienza idraulica del pozzo rappresenta al contempo uno strumento di notevole importanza e di straordinaria delicatezza. Il mancato raggiungimento della portata di progetto non può essere imputata di principio all'impresa e talvolta neanche al progettista. Una grave inefficienza idraulica (a prescindere dalla portata) è da ricondurre alle modalità costruttive; quindi prevalentemente le responsabilità sono dell'impresa e limitatamente posso essere ricondotte anche alle scelte progettuali. La DL ha quindi, a termini di norma, uno strumento forte per condizionare l'operato dell'impresa: strumento che però ha bisogno di essere usato con molto equilibrio.

Infatti le conoscenze scientifiche sullo specifico argomento non sono esaustive e le diverse procedure (dal metodo di Walton al T.I. adottato nella norma) aiutano sì a comprendere il 
fenomeno, ma non ne esauriscono la valenza. In particolare le condizioni di applicabilità dell'equazione di flusso, nel caso di un pozzo reale, non sono quasi mai applicabili in assoluto, anche se sono diffusamente accettate in approssimazione. Non sono accettabili, comunque, nei tanti pozzi reali realizzati in acquiferi fratturati o porosi con elevata variazione granulometrica o multistrato con piezometrie diverse.

La norma sulla qualificazione delle imprese di perforazione è stata completata e sotto esame UNI prima dell'inchiesta pubblica. La norma sulla qualificazione degli operatori è ancora in corso di elaborazione.

\section{Pozzi per acqua}

La norma per gli impianti a pompa di calore affronta alcuni aspetti importanti ma limitati in ordine al tema molto più ampio dei pozzi. Per questo motivo abbiamo promosso un gruppo di lavoro specifico per la definizione di Norme UNI per i pozzi.

I progetti di norma, al momento sono almeno due:

1. La progettazione dei pozzi per acqua

2. La costruzione dei pozzi per acqua

In realtà sarà necessario, in analogia alla norma sulla geotermia, una ulteriore norma sulla qualificazione delle imprese e degli operatori: tema per noi particolarmente sensibile.

In questo momento stiamo lavorando al primo progetto (ormai in fase conclusiva), successivamente inizieremo il secondo progetto, certamente meno delicato ma più corposo.

Dalle verifiche che abbiamo effettuato non risulta che in ambito internazionale esistano norme organiche per la progettazione dei pozzi: questo costituisce già un elemento di importante novità.

La norma è stata concepita "immaginando di prendere per mano un progettista inesperto e accompagnarlo fino ad esaurire tutti i compiti principali" che riguardano un'opera importante e complessa, anche se questa spesso assume un valore economico limitato. La norma può apparire talvolta didascalica, in essa sono contenute molte appendici informative che aiutano e orientano il progettista.

Fondamentale sarà, inoltre la compilazione delle schede di progettazione e dimensionamento. Queste aiuteranno il progettista in un percorso logico che si concluderà organizzando nel dettaglio i documenti progettuali indicati nell'ultimo capitolo della norma.

Ma è sull'aspetto dei contenuti che il gruppo di lavoro ma- nifesta particolare soddisfazione: si è cercato di costruire un percorso logico, consequenziale che partendo dal contesto geologico definisca i contenuti del progetto definitivo attraverso passaggi rigorosi, talvolta matematici, cercando di affrancarsi dalla prassi dell'approssimazione o dell'arbitrario ingiustificato che talvolta connota i progetti del nostro settore.

Scorrendo i titoli dei capitoli principali troviamo:

- Lanalisi per la progettazione, dove si sviluppa il contesto di progetto, la fattibilità geologica, la fattibilità ambientale, l'impatto della perforazione.

- La progettazione preliminare, sarà svolta definendo gli aspetti di salvaguardia ambientale, identificazione degli acquiferi, funzionalità ed efficienza dell'opera, affidabilità nel tempo ed economicità. Tutti questi argomenti saranno fissati con la compilazione di una scheda specifica predisposta fra gli allegati.

- La progettazione definitiva, che si articolerà nel dimensionamento dell'opera e sua verifica strutturale (con la compilazione della seconda scheda allegata), la scelta della tecnica di perforazione, la predisposizione delle opere di completamento, la manutenzione e la dismissione.

- I contenuti del documento progettuale, nel quale si elencano tutti gli elaborati necessari per una corretta progettazione.

Solo dall'elenco sintetico dei capitoli principali si comprende che il documento è abbastanza articolato e le trenta pagine di norma oltre alle quaranta di allegati ne danno la dimensione. Naturalmente sono previsti percorsi meno pesanti per le opere più semplici,

Riteniamo fondamentale un ampio approfondimento con tutti gli operatori interessati prima che il progetto diventi effettivamente Norma UNI.

A tal scopo abbiamo immaginato di organizzare una giornata per la presentazione e l'approfondimento dei contenuti del progetto nel mese di ottobre 2013 quando il lavoro sarà certamente completato, già sottoposto a UNI e speriamo in inchiesta pubblica.

Sarebbe anche interessante coinvolgere i nostri corrispondenti e amici europei di EGA, convinti come siamo che gli elementi di originalità di questo progetto possano essere di interesse anche per loro e magari diventare base di un progetto di norma europea che possa fregiarsi della denominazione UNI-EN. 